\title{
Nucleoside and nucleotide analogues by catalyst free Huisgen nitrile oxide-alkyne 1,3-dipolar cycloaddition $\uparrow$
}

\author{
Virginie Algay, Ishwar Singh and Frances Heaney* \\ Received 25th August 2009, Accepted 25th September 2009 \\ First published as an Advance Article on the web 28th October 2009 \\ DOI: $10.1039 / \mathrm{b} 917450 \mathrm{~h}$
}

An efficient, catalyst free, 1,3-dipolar cycloaddition strategy to conjugate nucleosides and nucleotides with isoxazoles under atmospheric conditions and in an aqueous environment is reported. The protocol involves chloramine- $\mathrm{T}$ as a practical reagent to induce in situ nitrile oxide formation and the alkyne partner is attached to the sugar residue or the nucleobase. The reactions are regiospecific, fast and high yielding.

\section{Introduction}

In the last 20 years or so non natural nucleic acid and nucleoside analogues have demonstrated significant clinical value as anticancer and antiviral compounds and many structural variations involving changes to the nucleobase and, or the ribofuranose moiety have been explored. ${ }^{1-4}$ In parallel, the isoxazole ring has been identified as attractive in terms of its hydrolytic stability, its potential for $\pi$-stacking and H-bonding and due to its sharing the isosteric character of the amide and ester bond. ${ }^{5,6}$ It is well recognised that the activities of different segments within a molecule can act in concert or confer new attributes on the molecule, and in this context the synthesis and bioactivity of isoxazole, isoxazoline and isoxazolidine modified nucleosides has received much attention. Several families of analogues have been explored, many involve heterocyclic replacement of the nucleoside ribose $^{7-11}$ whilst others fall into the class of $C^{5}$-modified pyrimidine nucleoside drugs, ${ }^{12,13}$ and others examine the influence of an $N^{3}$-tethered heterocycle, ${ }^{8,14}$ finally, isoxazoles have been introduced both directly and remotely to the $4^{\prime}$-ribose carbon. ${ }^{15,16}$ Two significant nucleoside derivatives include the $N^{I}$-tethered isoxazole 1 which has potent activity against the Polio virus ${ }^{7}$ and the $N^{3}$-ligated isoxazoline 2 which displays potent anti-HIV activity. ${ }^{14}$ Further interesting applications of heterocyclic modifications within nucleotide and oligonucleotide chemistry include isoxazole or isoxazoline replacement of the nucleotide phosphodiester linkage $\mathrm{e}^{17,18}$ and the recently reported nitrile oxide click modification of DNA by cycloaddition to alkynes ${ }^{19}$ and strained alkenes. ${ }^{20}$ In this context we wish to report an efficient synthetic approach to the preparation of sugar $\left(\mathrm{C}^{3^{\prime}}-\mathrm{O}-\right)$ and nucleobase $\left(\mathrm{N}^{3}-\right)$ tethered isoxazole thymidines. The key reaction, a nitrile oxide-alkyne click cycloaddition, proceeds selectively and in high yield under mild reaction conditions. That the protocol is free from copper is significant for broader applications as it removes the need to manage potentially problematic redox interference chemistry; thus, it presents a complementary approach to the azide-alkyne

Department of Chemistry, National University of Ireland, Maynooth, Co. Kildare, Republic of Ireland. E-mail: mary.f.heaney@nuim.ie; Fax: +353 1708 3815; Tel: +35317083802

$\dagger$ Electronic supplementary information (ESI) available: NMR spectra of compounds 4-16 and 20-22. See DOI: 10.1039/b917450h cycloaddition which has been highly successful in generation of nucleoside, nucleotide and oligonucleotide conjugates. ${ }^{21}$
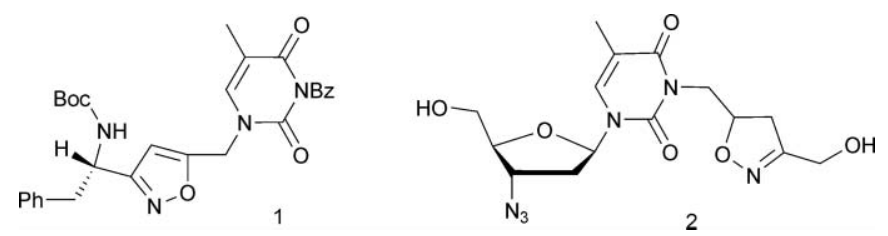

The preparation of isoxazole modified nucleosides by selection of an isoxazole carboxylic acid as one component of the Ugi reaction $^{16}$ or by cyclisation approaches ${ }^{22}$ represents alternatives to the commonly employed 1,3-dipolar cycloaddition route. The latter approach has been demonstrated with the nucleoside bearing either the alkyne moiety or the nitrile oxide precursor; both ribose $\mathrm{e}^{15,17}$ and nucleobase $\mathrm{e}^{13}$ modified oximes have served as nitrile oxide precursors, whilst the alkyne functionality is more commonly found on the base unit ${ }^{7,8,12}$ than on the sugar. ${ }^{22}$ In the reports to date $\mathrm{NaOCl}$, NCS or NBS have been selected as the dipole generating agents, generally the reactions are of 10-24 h duration and yields vary from fair to good. In one recent example a solid phase approach was demonstrated, ${ }^{8}$ though more generally the cycloadditions are reported in solution with THF or $\mathrm{CH}_{2} \mathrm{Cl}_{2}$ as reaction solvent.

We have recently shown solid phase nitrile oxide click cycloaddition chemistry to be successful for the preparation of isoxazole modified DNA ${ }^{19}$ and for generating $2^{\prime}$-OMe ribonucleoside and ribonucleotide analogues by [3+2]-cycloaddition to $2^{\prime}-O$-propargyl substrates. ${ }^{23}$ In this paper we further demonstrate the utility of this reaction in the solution phase synthesis of sugar $\left(C^{3^{\prime}}-O\right)$ and nucleobase $\left(N^{3}-\right)$ tethered isoxazoles.

\section{Results and discussion}

Nucleoside chemistry is delicate due to the numerous reactive sites and the first step in the synthetic sequence required selective protection of the $5^{\prime}-\mathrm{OH}$ of thymidine, thus, the already known $\mathbf{4}^{24}$ was prepared from thymidine $\mathbf{3}$ in good yield. Introduction of the $C^{3^{\prime}}$-O-propargyl group proceeded by nucleophilic displacement. Propargyl bromide was selected as the alkylating agent and $\mathrm{NaH}$ as the base. It was necessary to exercise caution during the addition of the bromide and, to ensure regioselective formation of $\mathbf{5}$, a 


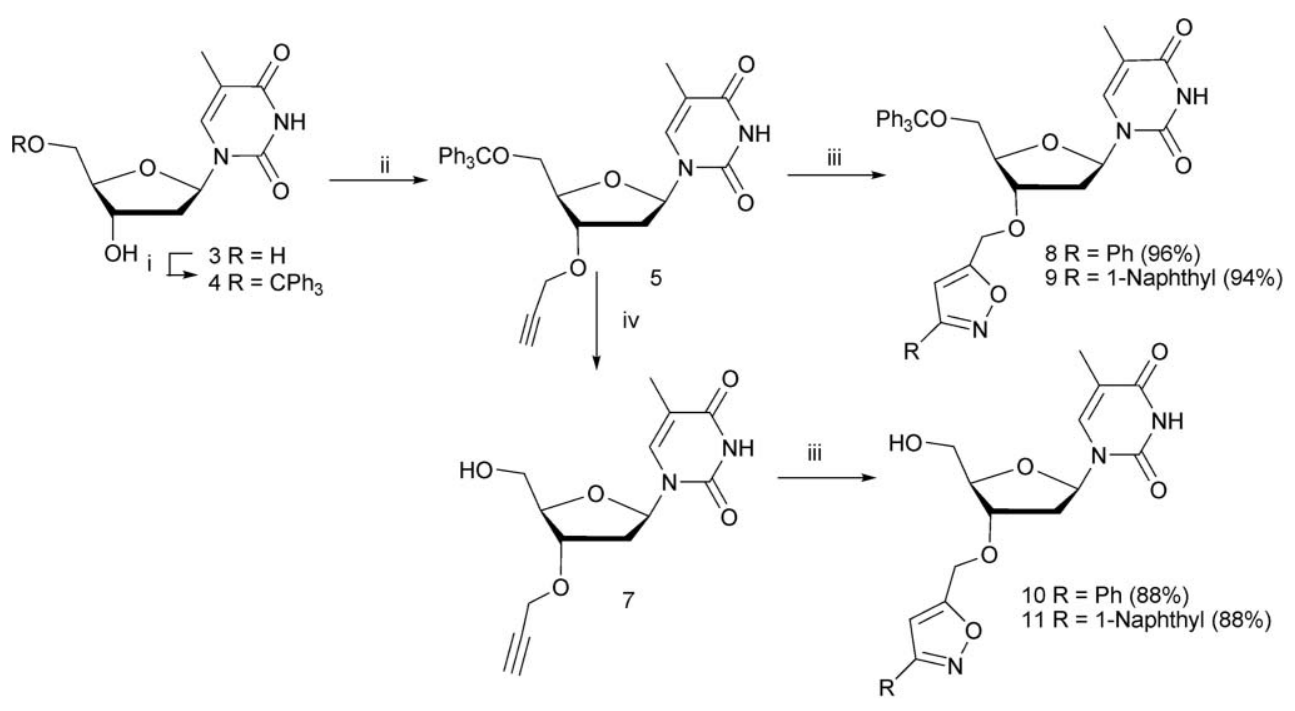

i. Anh. pyridine, $\mathrm{Ph}_{3} \mathrm{CCl}, \mathrm{Ar}, 0.5 \mathrm{~h}, 100{ }^{\circ} \mathrm{C}(86 \%)$; ii. Anh THF, $\mathrm{NaH}, \mathrm{Ar}, \mathrm{rt}, 1 \mathrm{~h}$; then $0{ }^{\circ} \mathrm{C}, \mathrm{CHCCH}_{2} \mathrm{Br}, 17 \mathrm{~h}, \mathrm{rt}(93 \%)$; iii. RCHNOH, Chloramine T, $\mathrm{NaHCO}_{3}$, EtOH, 1h, rt; iv.DCA, $\mathrm{CH}_{2} \mathrm{Cl}_{2}, \mathrm{rt}, 0.5 \mathrm{~h}(96 \%)$

Scheme 1

solution of this reagent in THF was added dropwise at $0{ }^{\circ} \mathrm{C}$; without this precaution the $C^{3^{\prime}}-O, N^{3}$-bisalkylated product 6 was found as a side product, Scheme 1. Most of the antiviral nucleoside analogues have 5'-hydroxyl groups for kinase phosphorylation, however, some $5^{\prime}$-tritylated derivatives have demonstrated inhibition of thymidine phosphorylase and angiogenesis. ${ }^{25}$ Thus, it was deemed important to explore the cycloaddition reaction with the 5 '-OH group both protected and unprotected, accordingly, DCA (dichloroacetic acid) mediated detritylation of $\mathbf{5}$ furnished the known 7.26

With the desired thymidine dipolarophiles 5 and 7 to hand the concept that chloramine-T mediated nitrile oxide-alkyne cycloaddition chemistry offers a tool to prepare nucleoside derivatives was tested. Aromatic appendages are interesting for biological activity, accordingly benzaldehyde oxime and 1-naphthaldehyde oxime were selected as the nitrile oxide precursors. The choice of chloramine- $\mathrm{T}$ as the dipole generating agent ${ }^{27}$ facilitates a move away from $\mathrm{CH}_{2} \mathrm{Cl}_{2}$ or THF and aqueous ethanolic $\mathrm{NaHCO}_{3}$ was the solvent of choice. The $5^{\prime}$-protected thymidine $\mathbf{5}$ was first examined. The reaction components were simply mixed together in aqueous ethanolic sodium hydrogen carbonate at room temperature. The reaction was very fast, reaching completion simply upon stirring for $1 \mathrm{~h}$ under atmospheric conditions and following purification 8 and 9 were isolated in $96 \%$ and $94 \%$ yield respectively. The reaction was equally successful with the 5 -deprotected substrate 7 , and the adducts from cycloaddition to benzonitrile oxide and 1-naphthonitrile oxide, $\mathbf{1 0}$ and $\mathbf{1 1}$ respectively, were both obtained in $88 \%$, Scheme 1 .

The structural elucidation of the cycloadducts was made on the basis of their NMR spectra. In all four cases an examination of the ${ }^{1} \mathrm{H}$ NMR spectra of the crude reaction products was suggestive of a high yielding, regiospecific formation of a 3,5disubstituted isoxazole. A singlet resonance in the region 6.52$6.62 \mathrm{ppm}$, diagnostic for the $4-H$ proton of the isoxazole ring, confirms the regiochemistry of the reaction; the expected position for the regioisomeric 3,4-disubstituted isoxazole would be $\sim 1 \mathrm{ppm}$ further downfield. ${ }^{28}$ The regioselectivity of the reaction is also supported by the presence of only one signal representing the $\mathrm{OCH}_{2}$-isoxazole methylene protons. These protons present as $\mathrm{AB}$ doublets at $\sim 4.6 \mathrm{ppm}$ whilst in the starting propargylated thymidines the corresponding protons appeared $\sim 0.5 \mathrm{ppm}$ upfield as part of a multiplet signal together with the resonance of the $4^{\prime}$-proton.

The $C^{3^{\prime}}-O-, N^{3}$-bispropargyl modified thymidine 6 was prepared in good yield from 4 following deprotonation with $\mathrm{NaH}$ (6 eq.) and alkylation with propargyl bromide (3 eq.). The successful formation of the diynyl nucleoside was evident from the appearance of two triplet resonances in the ${ }^{1} \mathrm{H}$ NMR spectrum at 2.41 and $2.17 \mathrm{ppm}$, diagnostic of the inequivalent alkyne protons; the adjacent methylene protons appeared as doublets at $4.25\left(\mathrm{OCH}_{2}\right)$ and $4.77\left(\mathrm{NCH}_{2}\right)$ ppm respectively. Both the 5'tritylated diynyl 6 and its $5^{\prime}$-hydroxy parent 12 succumbed to the nitrile oxide click cycloaddition protocol described above. Thus, the aryl nitrile oxides generated from reaction of benzaldehyde oxime or 1-naphthaldehyde oxime with chloramine-T reacted, in turn, with $\mathbf{6}$ or $\mathbf{1 2}$ under atmospheric conditions, at ambient temperature in ethanolic sodium hydrogen carbonate. No compromise in regioselectivity was noted in formation of the double cycloaddition products $\mathbf{1 3}$ through $\mathbf{1 6}$ which were isolated in 78$87 \%$ yield, Scheme 2. Once again ${ }^{1} \mathrm{H}$ NMR spectral evidence supports formation of the 3,5-disubstituted isoxazoles; in all cases, concomitant with cycloaddition was the disappearance of the alkyne proton resonances and a downfield shift $(\sim 0.5 \mathrm{ppm})$ in the position of the both the $\mathrm{NCH}_{2}$ and the $\mathrm{OCH}_{2}$ methylene protons. No other regioisomer could be found amongst the crude products.

To show compatibility with the nucleotide phosphodiester backbone 20 was prepared by coupling of the alkynyl alcohol $17^{19}$ to the $5^{\prime}$-Dmt protected thymidine phosphoramidite 18. Following oxidation and work-up, the protecting group was removed from 19 by treatment with DCA and 20 was obtained in 53\% yield over the two steps, Scheme 3 . Analysis by ${ }^{31} \mathrm{P},{ }^{13} \mathrm{C}$ and ${ }^{1} \mathrm{H}$ NMR spectroscopy together with HRMS data confirmed the expected 


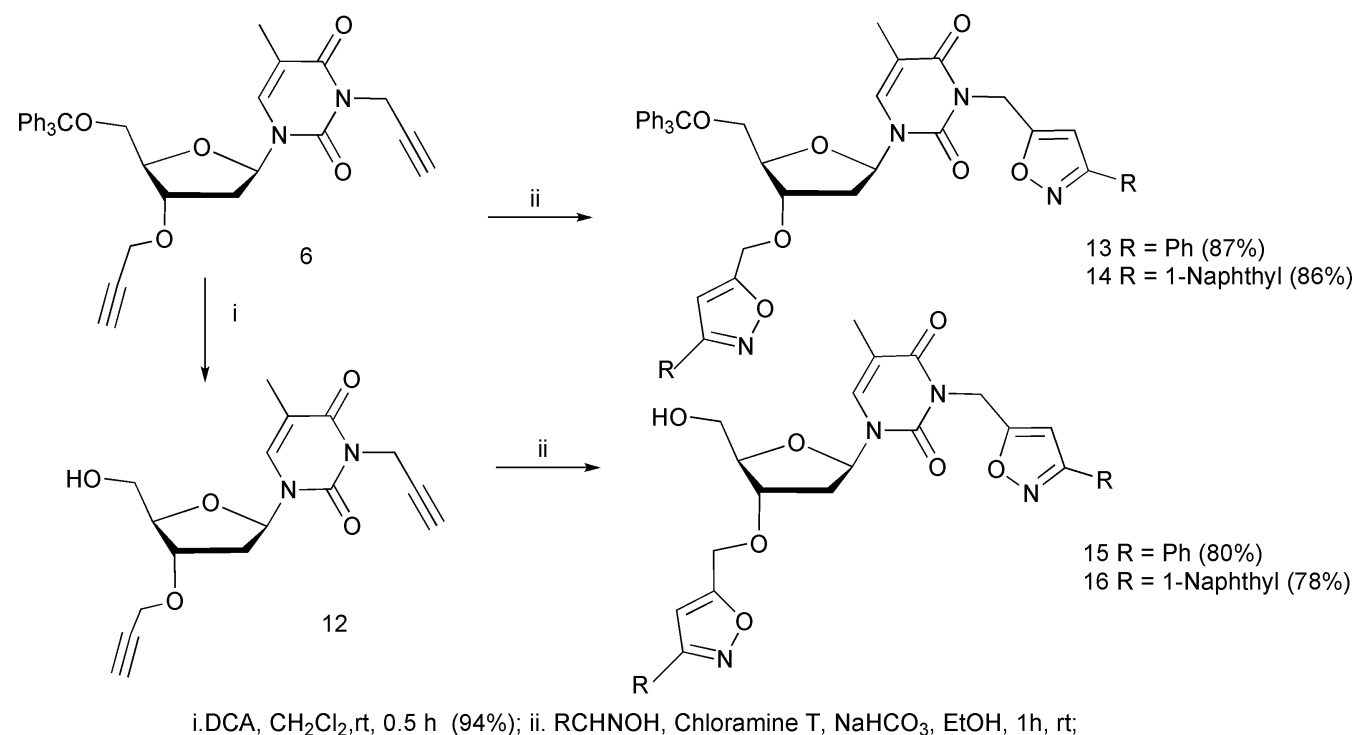

Scheme 2

structure. In particular a resonance at $\delta-2.6 \mathrm{ppm}$ is diagnostic for the $\mathrm{P}(\mathrm{v})$ nucleus; proton and ${ }^{13} \mathrm{C}$ assignments were supported by 2D-COSY experiments.

The nucleotide 20 subjected to the cycloaddition protocol under the same conditions as described above for the nucleoside substrates. Thus the phenyl and naphthyl substituted isoxazole derivitized nucleotides $\mathbf{2 1}$ and $\mathbf{2 2}$ were obtained in $77 \%$ and $72 \%$ yield respectively following reaction with the appropriate oxime and chloramine-T in ethanolic $\mathrm{NaHCO}_{3}(\mathrm{rt}, 1 \mathrm{~h})$, Scheme 3 . Characterisation of the cycloadducts was made on the basis of their NMR spectral data. In both cases a singlet resonance in the region 6.58-6.59 ppm, diagnostic for the $4-H$ proton of the isoxazole ring confirms formation of a 3,5-disubstituted isoxazole, further confirmation of the regiochemistry of the reaction is supported by the appearance of the $\mathrm{OCH}_{2}$ isoxazole protons as $\mathrm{AB}$ doublets at $\sim 4.7 \mathrm{ppm}$.

\section{Conclusion}

In conclusion nitrile oxide-alkyne click cycloaddition chemistry offers a robust route to novel isoxazole conjugated nucleosides or nucleotides with either $5^{\prime}$-trityl protection or with a free $5^{\prime}-\mathrm{OH}$ available for additional structural elaboration. The ability to incorporate an isoxazole nucleus in the design of new thymidine receptor agonists is potentially very valuable and the significance of the current work lies in the demonstration of a high yielding chloramine-T protocol for rapid, catalyst free nucleoside and nucleotide elaboration under very mild conditions viz room

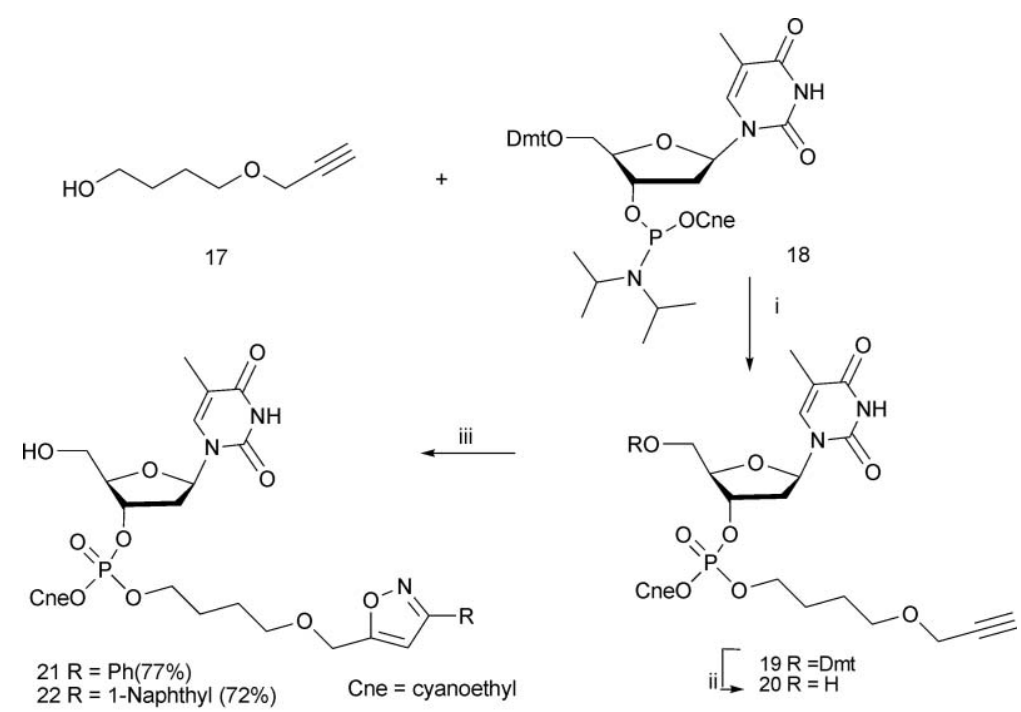

i. Anh $\mathrm{CH}_{3} \mathrm{CN}, \mathrm{BMT}, \mathrm{Ar}, 0.5 \mathrm{~h}$, rt; then $\mathrm{I}_{2}$, THF, pyridine, $\mathrm{H}_{2} \mathrm{O}, 0.5 \mathrm{~h}, 0^{\circ} \mathrm{C}$

ii. DCA, $\mathrm{CH}_{2} \mathrm{Cl}_{2}, \mathrm{rt}, 0.5 \mathrm{~h}$; iii. $\mathrm{RCHNOH}$, Chloramine T, $\mathrm{NaHCO}_{3}, \mathrm{EtOH}, 1 \mathrm{~h}$, rt 
temperature, atmospheric conditions and an aqueous environment. The synthetic utility of the nitrile oxide reaction, which reaches completion in $60 \mathrm{~min}$ at $\mathrm{rt}$, compares favourably with the observed copper catalysed formation of triazole linked dithymidines which whilst complete within 3 min under microwave activation $\left(80{ }^{\circ} \mathrm{C}, 300 \mathrm{~W}\right),{ }^{29}$ requires $24 \mathrm{~h}$ to reach $\sim 40 \%$ completion at $\mathrm{rt}^{30}$

\section{Experimental}

Analytical TLC was performed on precoated $(250 \mu \mathrm{m})$ silica gel 60 F-254 plates from Merck. All plates were visualized by UV irradiation, and/or staining with $5 \% \mathrm{H}_{2} \mathrm{SO}_{4}$ in ethanol, followed by heating. Flash chromatography grade silica gel $60(230-400$ mesh) was obtained from Merck. Mass analysis was performed on a LC/TOF-MS instrument. The LC was a model 1200 Series (Agilent Corp, Santa Clara, CA), injection volume was $10 \mu \mathrm{L}$, the column was an Agilent Eclipse XBD-C18 (Agilent), 5-micron with a mobile phase of $\mathrm{A}=\mathrm{ACN}$ with $0.1 \%$ formic acid and $\mathrm{B}=0.1 \%$ formic acid in water. The gradient was $5 \%$ to $100 \%$ A over $15 \mathrm{~min}$ at a flow rate of $0.5 \mathrm{~mL} \mathrm{~min}^{-1}$. The LC/TOFMS was a model 6210 Time-Of-Flight LC/MS (Agilent Corp, Santa Clara, CA) with an electrospray source positive and negative (ESI \pm ), capilary 3,500 V, nebuliser spray 30 psig, drying gas $5 \mathrm{~L} \mathrm{~min}^{-1}$, source temperature $325^{\circ} \mathrm{C}$. The fragmentor was used at $175 \mathrm{~V}$. Reference masses (Agilent solution) were 121.050873, 149.02332, 322.048121, 922.009798, 1221.990633, 1521.971475, and $2421.91399 \mathrm{~m} / \mathrm{z}$. The NMR spectra were obtained for ${ }^{1} \mathrm{H}$ at $300 \mathrm{MHz}$, for ${ }^{13} \mathrm{C}$ at $75 \mathrm{MHz}$ and for ${ }^{31} \mathrm{P}$ at $121 \mathrm{MHz}$ on a Bruker instrument at $25^{\circ} \mathrm{C}$. All samples for NMR were prepared in $\mathrm{CDCl}_{3}$. Chemical shifts are reported in ppm downfield from TMS as standard and coupling constants are reported in hertz. Chemical agents were purchased from Aldrich Chemical Company unless otherwise noted.

\section{3'-O-(2-Propynyl)-5'-O-(triphenylmethyl)thymidine 5}

To a solution of $5^{\prime}$ - $O$-trityl-3'-O-propargylthymidine 4 (100 mg, $0.206 \mathrm{mmol})$ in anhydrous THF $(4 \mathrm{~mL})$ at $0{ }^{\circ} \mathrm{C}$ was added slowly, under argon, anhydrous sodium hydride (13 $\mathrm{mg}, 0.516 \mathrm{mmol})$ and the mixture was stirred at RT for $1 \mathrm{~h}$. The reaction mixture was cooled to $0{ }^{\circ} \mathrm{C}$ prior to the addition, drop by drop, of a solution of propargyl bromide $(0.206 \mathrm{mmol})$ in THF $(1 \mathrm{~mL})$. The mixture was allowed to slowly warm to RT and stirred for a further $17 \mathrm{~h}$. The reaction was quenched by addition of water. The product was isolated following extraction with ethyl acetate. The organic layer was dried over anhydrous sodium sulfate and the solvent was removed under reduced pressure. The crude product was purified by flash column chromatography over silica gel using dichloromethane and acetone $(95: 5)$ as eluant to give the title compound as an off-white foam $(93 \%)$.

$R_{\mathrm{f}} 0.31$ (DCM, Acetone, $\left.95: 5\right) ;{ }^{1} \mathrm{H}$ NMR: $\delta 8.88(1 \mathrm{H}$, br s, NH), 7.58 (1H, s, H6), 7.50-7.18 (15H, m, Ph), 6.37-6.30 (1H, m, H1'), 4.56-4.49 (1H, m, H3'), 4.19-4.11 (3H, m, H4', $\left.\mathrm{OCH}_{2}\right), 3.47(1 \mathrm{H}$, $\left.\mathrm{dd},{ }^{2} J 10.6,{ }^{3} J 3.0, \mathrm{H} 5^{\prime}\right), 3.38\left(1 \mathrm{H}, \mathrm{dd},{ }^{2} J 10.6,{ }^{3} J 3.0, \mathrm{H} 5^{\prime}\right), 2.58$ $2.47\left(1 \mathrm{H}, \mathrm{m}, \mathrm{H} 2^{\prime}\right), 2.41\left(1 \mathrm{H}, \mathrm{t},{ }^{4} \mathrm{~J} 2.4, \mathrm{C} \equiv \mathrm{CH}\right), 2.29-2.19(1 \mathrm{H}, \mathrm{m}$, $\left.\mathrm{H} 2^{\prime}\right), 1.49\left(3 \mathrm{H}, \mathrm{s}, \mathrm{CH}_{3}\right) ;{ }^{13} \mathrm{C} \mathrm{NMR}: \delta 163.7\left(\mathrm{C}_{2}\right), 150.3\left(\mathrm{C}_{4}\right), 143.4$ (ArC), $135.4\left(\mathrm{C}_{6}\right), 128.7$ (ArC), 128.1 (ArC), 127.4 (ArC), 111.2 $\left(\mathrm{C}_{5}\right), 87.5\left(\mathrm{CPh}_{3}\right), 84.8\left(\mathrm{C}_{1^{\prime}}\right), 83.8\left(\mathrm{C}_{4^{\prime}}\right), 79.1(\mathrm{C} \equiv \mathrm{CH}), 78.5\left(\mathrm{C}_{3^{\prime}}\right)$,
$75.1(\mathrm{CH} \equiv \mathrm{C}), 63.6\left(\mathrm{C}_{5^{\prime}}\right), 53.4\left(\mathrm{OCH}_{2}\right), 37.8\left(\mathrm{C}_{2^{\prime}}\right), 11.9\left(\mathrm{CH}_{3}\right)$; HRMS (ESI): $m / z \quad \mathrm{C}_{32} \mathrm{H}_{30} \mathrm{~N}_{2} \mathrm{NaO}_{5}$ requires $545.2047[\mathrm{M}+\mathrm{Na}]^{+}$, found $545.2052(0.96 \mathrm{ppm})$.

\section{General procedure for the nitrile oxide-alkyne cycloaddition}

To a round bottomed flask containing the oxime ( 2 eq. for the monoalkynes 5, 7 and 19 or 3 eq. for the bisalkynes 6 and 12) and chloramine-T monohydrate $(2.5$ eq. for the mono- or 3.5 eq. for the bisalkynes) in $4 \%$ aqueous sodium hydrogen carbonate $(2 \mathrm{~mL}$ per $0.1 \mathrm{mmol}$ of oxime) and ethanol ( $1 \mathrm{~mL}$ per $0.1 \mathrm{mmol}$ oxime) was added the propargylated thymidine. The mixture was stirred for $1 \mathrm{~h}$ at RT after which analysis by TLC indicated complete reaction. The product was isolated following extraction with ethyl acetate. The organic layer was dried over anhydrous sodium sulfate and the solvent was removed under reduced pressure to afford the crude products which were purified as described below.

\section{$3^{\prime}-O$-[(3-Phenylisoxazol-5-yl)methyl] $]-5$ - $O$ - (triphenylmethyl)thymidine 8}

The crude product was purified by flash column chromatography over silica gel, eluting with dichloromethane and acetone $(97: 3)$, to give the title compound as an off-white solid, (96\%).

$R_{\mathrm{f}} 0.46$ (DCM, Acetone, $\left.95: 5\right) ;{ }^{1} \mathrm{H}$ NMR: $\delta 8.78(1 \mathrm{H}$, br s, NH), $7.83-7.18(21 \mathrm{H}, \mathrm{m}, \mathrm{H} 6, \mathrm{Ph}), 6.52(1 \mathrm{H}, \mathrm{s}, \mathrm{CH}=\mathrm{CO}$ iso $), 6.41-6.34$ $\left(1 \mathrm{H}, \mathrm{m}, \mathrm{H} 1^{\prime}\right), 4.65\left(1 \mathrm{H}, \mathrm{d},{ }^{2} J 13.9, \mathrm{OCH}_{2}\right), 4.58\left(1 \mathrm{H}, \mathrm{d},{ }^{2} J 13.9\right.$, $\left.\mathrm{OCH}_{2}\right), 4.38-4.32\left(1 \mathrm{H}, \mathrm{m}, \mathrm{H} 3^{\prime}\right), 4.21-4.16\left(1 \mathrm{H}, \mathrm{m}, \mathrm{H} 4^{\prime}\right), 3.50(1 \mathrm{H}$, $\left.\mathrm{dd},{ }^{2} J 10.6,{ }^{3} J 3.3, \mathrm{H} 5^{\prime}\right), 3.35\left(1 \mathrm{H}, \mathrm{dd},{ }^{2} J 10.6,{ }^{3} J 2.7, \mathrm{H} 5^{\prime}\right), 2.59$ $2.49\left(1 \mathrm{H}, \mathrm{m}, \mathrm{H} 2^{\prime}\right), 2.31-2.18\left(1 \mathrm{H}, \mathrm{m}, \mathrm{H} 2^{\prime}\right), 1.52\left(3 \mathrm{H}, \mathrm{s}, \mathrm{CH}_{3}\right) ;{ }^{13} \mathrm{C}$ NMR: $\delta 169.0(\mathrm{C}=\mathrm{N}$ iso $), 163.7\left(\mathrm{C}_{2}\right), 162.5(\mathrm{CO}=\mathrm{CH}$ iso $), 150.3$ $\left(\mathrm{C}_{4}\right), 143.2$ (ArC), $135.3\left(\mathrm{C}_{6}\right), 130.2$ (ArC), 129.0 (ArC), 128.7 (ArC), 128.6 (ArC), 128.1 (ArC), 127.5 (ArC), 126.9 (ArC), 111.4 $\left(\mathrm{C}_{5}\right), 101.3(\mathrm{CH}=\mathrm{CO}$ iso $), 87.6\left(\mathrm{CPh}_{3}\right), 84.8\left(\mathrm{C}_{1^{\prime}}\right), 83.8\left(\mathrm{C}_{4^{\prime}}\right), 80.1$ $\left(\mathrm{C}_{3^{\prime}}\right), 63.8\left(\mathrm{C}_{5^{\prime}}\right), 62.2\left(\mathrm{OCH}_{2}\right), 37.8\left(\mathrm{C}_{2^{\prime}}\right), 11.9\left(\mathrm{CH}_{3}\right)$; HRMS (ESI): $m / z \mathrm{C}_{39} \mathrm{H}_{35} \mathrm{~N}_{3} \mathrm{NaO}_{6}$ requires $664.2418[\mathrm{M}+\mathrm{Na}]^{+}$, found 664.2398 (-3.07 ppm).

\section{$3^{\prime}-O$-[3-[(1-Naphthyl)isoxazol-5-yl]methyl]-5'-O- (triphenylmethyl)thymidine 9}

The crude product was purified by flash column chromatography over silica gel using dichloromethane and acetone $(95: 5)$ as eluant to give the title compound as an off-white solid (94\%).

$R_{\mathrm{f}} 0.21$ (DCM, Acetone, 95:5); ${ }^{1} \mathrm{H}$ NMR: $\delta 8.41(1 \mathrm{H}$, br s, $\mathrm{NH}), 8.38-7.17$ (23H, m, H6, Ph, Naph), 6.53 (1H, s, CH=CO iso), 6.42-6.35 (1H, m, H1'), $4.73\left(1 \mathrm{H}, \mathrm{d},{ }^{2} J 13.6, \mathrm{OCH}_{2}\right), 4.65$ $\left(1 \mathrm{H}, \mathrm{d},{ }^{2} J 13.6, \mathrm{OCH}_{2}\right), 4.45-4.38\left(1 \mathrm{H}, \mathrm{m}, \mathrm{H} 3^{\prime}\right), 4.24-17(1 \mathrm{H}$, m, H4'), $3.52\left(1 \mathrm{H}, \mathrm{dd},{ }^{2} J 10.7,{ }^{3} J 3.3, \mathrm{H}^{\prime}\right), 3.37\left(1 \mathrm{H}, \mathrm{dd},{ }^{2} J 10.6\right.$, ${ }^{3} J$ 3.0, H5'), 2.65-2.51 (1H, m, H2'), 2.34-2.21 (1H, m, H2'), 1.52 $\left(3 \mathrm{H}, \mathrm{s}, \mathrm{CH}_{3}\right) ;{ }^{13} \mathrm{C}$ NMR: $\delta 168.3(\mathrm{C}=\mathrm{N}$ iso $), 163.4\left(\mathrm{C}_{2}\right), 162.6$ $(C \mathrm{O}=\mathrm{CH}$ iso $), 150.2\left(\mathrm{C}_{4}\right), 143.2(\mathrm{ArC}), 135.3\left(\mathrm{C}_{6}\right),[133.8,130.9$, 130.4, 128.6, 128.5, 128.1, 127.9, 127.5, 127.1, 126.5, 126.3, 125.6, 125.2] ( $\mathrm{ArC}), 111.3\left(\mathrm{C}_{5}\right), 104.6(\mathrm{CH}=\mathrm{CO}$ iso $), 87.6\left(\mathrm{CPh}_{3}\right), 84.8$ $\left(\mathrm{C}_{1^{\prime}}\right), 83.8\left(\mathrm{C}_{4^{\prime}}\right), 80.2\left(\mathrm{C}_{3^{\prime}}\right), 63.8\left(\mathrm{C}_{5^{\prime}}\right), 62.3\left(\mathrm{OCH}_{2}\right), 37.9\left(\mathrm{C}_{2^{\prime}}\right), 11.9$ $\left(\mathrm{CH}_{3}\right)$; HRMS (ESI): $m / z \mathrm{C}_{43} \mathrm{H}_{38} \mathrm{~N}_{3} \mathrm{O}_{6}$ requires $692.2755[\mathrm{M}+\mathrm{H}]^{+}$, found 692.2741 (-2.1 ppm). 


\section{General procedure for deprotection of $5^{\prime}$-trityl thymidine derivatives}

The tritylated thymidine was stirred for $0.5 \mathrm{~h}$ at RT in a solution of DCA $3.5 \%$ in DCM $(1 \mathrm{~mL} / 0.1 \mathrm{mmol})$. The reaction mixture was washed with a saturated solution of sodium hydrogen carbonate until the aqueous layer became neutral. The organic layer was dried over anhydrous sodium sulfate and the solvent was removed under reduced pressure to afford the crude products which were purified as described below.

\section{$3^{\prime}$ - $O$-(2-Propynyl)thymidine $7^{25}$}

The crude product was purified by flash column chromatography over silica gel, eluting with dichloromethane and methanol (0 to $5 \%$ ), to give the title compound as an off-white foam $(96 \%)$.

$R_{\mathrm{f}} 0.18$ (DCM, MeOH, $\left.95: 5\right) ;{ }^{1} \mathrm{H}$ NMR: $\delta 9.45(1 \mathrm{H}$, br s, NH), 7.44 (1H, s, H6), $6.12\left(1 \mathrm{H}, \mathrm{t},{ }^{3} J\right.$ 6.6, H1'), 4.46-4.38 (1H, m, H3'), 4.29-4.09 (3H, m, H4', $\left.\mathrm{OCH}_{2}\right), 3.95\left(1 \mathrm{H}, \mathrm{dd},{ }^{2} J 12.1,{ }^{3} J 2.7, \mathrm{H}^{\prime}\right)$, $3.82\left(1 \mathrm{H}, \mathrm{dd},{ }^{2} J 12.1,{ }^{3} J\right.$ 3.0, H5'), 3.58 (1H, br s, OH), 2.49 $\left(1 \mathrm{H}, \mathrm{t},{ }^{4} \mathrm{~J} 2.4, \mathrm{C} \equiv \mathrm{CH}\right), 2.46-2.30\left(2 \mathrm{H}, \mathrm{m}, \mathrm{H} 2{ }^{\prime}\right), 1.90\left(3 \mathrm{H}, \mathrm{s}, \mathrm{CH}_{3}\right)$; HRMS (ESI): $m / z \mathrm{C}_{13} \mathrm{H}_{16} \mathrm{~N}_{2} \mathrm{NaO}_{5}$ requires $280.1148[\mathrm{M}+\mathrm{Na}]^{+}$, found 280.1178 (2.61 ppm).

\section{3'-O-[(3-Phenylisoxazol-5-yl)methyl]thymidine 10}

The crude product was purified by flash column chromatography over silica gel, eluting with dichloromethane and methanol (95:5), to give the title compound as an off-white solid (88\%).

${ }^{1} \mathrm{H}$ NMR: $\delta 8.55(1 \mathrm{H}$, br s, NH), 7.81-7.78 (2H, m, ArH), 7.46-7.44 (3H, m, ArH), $7.38(1 \mathrm{H}, \mathrm{s}, \mathrm{H} 6), 6.59(1 \mathrm{H}, \mathrm{s}, \mathrm{CH}=\mathrm{CO}$ iso)), $6.13\left(1 \mathrm{H}, \mathrm{t}, J 7.2, \mathrm{H}^{\prime}\right), 4.72\left(1 \mathrm{H}, \mathrm{d},{ }^{2} J 13.8, \mathrm{OCH}_{2}\right), 4.66$ $\left(1 \mathrm{H}, \mathrm{d},{ }^{2} J 13.8, \mathrm{OCH}_{2}\right), 4.40-4.37\left(1 \mathrm{H}, \mathrm{m}, \mathrm{H} 3{ }^{\prime}\right), 4.15(1 \mathrm{H} \mathrm{d}, J$ 2.7, $\left.\mathrm{H} 4{ }^{\prime}\right), 3.92\left(1 \mathrm{H} \mathrm{dd},{ }^{2} J 11.7,{ }^{3} J 2.7, \mathrm{H} 5^{\prime}\right), 3.80\left(1 \mathrm{H} \mathrm{dd},{ }^{2} J\right.$ 11.7, $\left.{ }^{3} \mathrm{~J} 3.0, \mathrm{H}^{\prime}\right), 2.41-2.37\left(2 \mathrm{H}, \mathrm{m}, \mathrm{H} 2{ }^{\prime}\right), 1.89\left(3 \mathrm{H}, \mathrm{s}, \mathrm{CH}_{3}\right) ;{ }^{13} \mathrm{C}$ NMR: $\delta 170.0(\mathrm{C}=\mathrm{N}$ iso $), 163.3\left(\mathrm{C}_{2}\right), 162.1(\mathrm{CO}=\mathrm{CH}$ iso $), 150.5$ $\left(\mathrm{C}_{4}\right), 136.0\left(\mathrm{C}_{6}\right), 130.0-126.6(\mathrm{ArH}), 109.9\left(\mathrm{C}_{5}\right), 101.2(\mathrm{CH}=\mathrm{CO}$ iso), $85.0\left(\mathrm{C}_{1^{\prime}}\right), 85.8\left(\mathrm{C}_{4^{\prime}}\right), 80.3\left(\mathrm{C}_{3^{\prime}}\right), 62.1\left(\mathrm{C}_{5^{\prime}}\right), 61.7\left(\mathrm{OCH}_{2}\right), 36.9$ $\left(\mathrm{C}_{2^{\prime}}\right), 11.6\left(\mathrm{CH}_{3}\right)$; HRMS (ESI): $m / z \mathrm{C}_{20} \mathrm{H}_{22} \mathrm{~N}_{3} \mathrm{O}_{6}$ requires 400.1509 $[\mathrm{M}+\mathrm{Na}]^{+}$, found $400.1494(1.16 \mathrm{ppm})$.

\section{3'-O-[3-[(1-Naphthyl)isoxazol-5-yl]methyl]thymidine 11}

The crude product was purified by flash column chromatography over silica gel, eluting with dichloromethane and methanol (95:5), to give the title compound as an off-white solid (88\%).

$R_{\mathrm{f}} 0.24$ (DCM, MeOH, 95:5); ${ }^{1} \mathrm{H}$ NMR: $\delta 8.39-8.32(1 \mathrm{H}, \mathrm{m}$, ArH), 7.99-7.89 (2H, m, ArH), 7.72-7.67 (1H, m, ArH), 7.60$7.50(3 \mathrm{H}, \mathrm{m}, \mathrm{ArH}), 7.38(1 \mathrm{H}, \mathrm{s}, \mathrm{H} 6), 6.62(1 \mathrm{H}, \mathrm{s}, \mathrm{CH}=\mathrm{CO}$ iso), $6.14\left(1 \mathrm{H}, \mathrm{t},{ }^{3} J 9.9, \mathrm{H}^{\prime}\right), 4.78\left(1 \mathrm{H}, \mathrm{d},{ }^{2} J 13.9, \mathrm{OCH}_{2}\right), 4.73(1 \mathrm{H}$, $\left.\mathrm{d},{ }^{2} \mathrm{~J} 13.9, \mathrm{OCH}_{2}\right), 4.49-4.41\left(1 \mathrm{H}, \mathrm{m}, \mathrm{H} 3^{\prime}\right), 4.23-4.16(1 \mathrm{H}, \mathrm{m}$, $\left.\mathrm{H} 4^{\prime}\right), 3.96$ (1H, dd, $\left.{ }^{2} J 12.5,{ }^{3} J 3.3, \mathrm{H}^{\prime}\right), 3.83\left(1 \mathrm{H}, \mathrm{dd},{ }^{2} J 12.4,{ }^{3} J\right.$ 2.7, H5'), 2.49-2.40 (2H, m, H2'), $1.92\left(3 \mathrm{H}, \mathrm{s}, \mathrm{CH}_{3}\right) ;{ }^{13} \mathrm{C} \mathrm{NMR}: \delta$ $168.3(\mathrm{C}=\mathrm{N}$ iso $), 163.5\left(\mathrm{C}_{2}\right), 162.7(\mathrm{CO}=\mathrm{CH}$ iso $), 150.3\left(\mathrm{C}_{4}\right), 137.0$ $\left(\mathrm{C}_{6}\right),[133.8,130.9,130.4,128.6,127.9,127.2,126.4,125.5,125.2]$ ( $\mathrm{ArC}), 111.4\left(\mathrm{C}_{5}\right), 104.8(\mathrm{CH}=\mathrm{CO}$ iso $), 87.5\left(\mathrm{C}_{1^{\prime}}\right), 85.0\left(\mathrm{C}_{4^{\prime}}\right), 80.0$ $\left(\mathrm{C}_{3^{\prime}}\right), 62.7\left(\mathrm{C}_{5^{\prime}}\right), 62.5\left(\mathrm{OCH}_{2}\right), 37.0\left(\mathrm{C}_{2^{\prime}}\right), 12.5\left(\mathrm{CH}_{3}\right)$; HRMS (ESI): $m / z \quad \mathrm{C}_{24} \mathrm{H}_{23} \mathrm{KN}_{3} \mathrm{O}_{6}$ requires $488.1218[\mathrm{M}+\mathrm{K}]^{+}$, found 488.1221 (0.58 ppm).
3-(2-Propynyl)-3'-O-(2-propynyl)-5'-O(triphenylmethyl)thymidine 6

To a solution of $5^{\prime}$ - $O$-trityl-3'- $O$-propargylthymidine 4 (195 mg, $0.402 \mathrm{mmol})$ in anhydrous $\mathrm{THF}(4 \mathrm{~mL})$ at $0{ }^{\circ} \mathrm{C}$ was added slowly, under argon, anhydrous sodium hydride (58 $\mathrm{mg}, 2.415 \mathrm{mmol})$ and the mixture was stirred at RT for $1 \mathrm{~h}$. Propargyl bromide $(1.40 \mathrm{~mL}$, $1.335 \mathrm{mmol}$ ) was added drop by drop and the mixture was stirred for $17 \mathrm{~h}$ at RT. The reaction was quenched by addition of water. The product was isolated following extraction with ethyl acetate. The organic layer was dried over anhydrous sodium sulfate and the solvent was removed under reduced pressure. The crude product was purified by flash column chromatography over silica gel using dichloromethane and acetone ( 0 to $2 \%)$ as eluant to give the title compound as an off-white foam (77\%).

$R_{\mathrm{f}} 0.58$ (DCM, Acetone, $\left.98: 2\right) ;{ }^{1} \mathrm{H}$ NMR: $\delta 7.62(1 \mathrm{H}, \mathrm{s}, \mathrm{H} 6)$, 7.50-7.20 (15H, m, Ph), 6.42-6.34 (1H, m, H1'), $4.77\left(2 \mathrm{H}, \mathrm{d},{ }^{4} J 2.4\right.$, $\left.\mathrm{NCH}_{2}\right), 4.58-4.49\left(1 \mathrm{H}, \mathrm{m}, \mathrm{H} 3^{\prime}\right), 4.25-4.07\left(3 \mathrm{H}, \mathrm{m}, \mathrm{H}^{\prime}, \mathrm{OCH}_{2}\right)$, $3.47\left(1 \mathrm{H}, \mathrm{dd},{ }^{2} J 10.7,{ }^{3} J 3.0, \mathrm{H} 5^{\prime}\right), 3.38\left(1 \mathrm{H}, \mathrm{dd},{ }^{2} J 10.7,{ }^{3} J 3.0\right.$, $\left.\mathrm{H}^{\prime}\right), 2.61-2.50\left(1 \mathrm{H}, \mathrm{m}, \mathrm{H} 2^{\prime}\right), 2.41\left(1 \mathrm{H}, \mathrm{t},{ }^{4} \mathrm{~J} 2.4, \mathrm{OCH}_{2} \mathrm{C} \equiv \mathrm{CH}\right)$, 2.32-2.19 (1H, m, H2'), $2.17\left(1 \mathrm{H}, \mathrm{t},{ }^{4} \mathrm{~J} 2.4, \mathrm{NCH}_{2} \mathrm{C} \equiv \mathrm{CH}\right), 1.51$ $\left(3 \mathrm{H}, \mathrm{s}, \mathrm{CH}_{3}\right) ;{ }^{13} \mathrm{C} \mathrm{NMR}: \delta 162.5\left(\mathrm{C}_{2}\right), 150.2\left(\mathrm{C}_{4}\right), 143.3(\mathrm{ArC}), 133.9$ $\left(\mathrm{C}_{6}\right),[128.7,128.9,128.7,128.1,127.9,127.5,127.1](\mathrm{ArC}), 110.1$ $\left(\mathrm{C}_{5}\right), 87.5\left(\mathrm{CPh}_{3}\right), 85.5\left(\mathrm{C}_{1^{\prime}}\right), 83.8\left(\mathrm{C}_{4^{\prime}}\right), 79.1\left(\mathrm{OCH}_{2} \mathrm{C} \equiv \mathrm{CH}\right), 78.3$ $\left(\mathrm{NCH}_{2} \mathrm{C} \equiv \mathrm{CH}, \mathrm{C}_{3^{\prime}}\right), 75.1\left(\mathrm{OCH}_{2} \mathrm{C} \equiv \mathrm{CH}\right), 70.6\left(\mathrm{NCH}_{2} \mathrm{C} \equiv \mathrm{CH}\right)$, $63.6\left(\mathrm{C}_{5^{\prime}}\right), 56.7\left(\mathrm{OCH}_{2}\right), 37.9\left(\mathrm{C}_{2^{\prime}}\right), 30.4\left(\mathrm{NCH}_{2}\right), 12.6\left(\mathrm{CH}_{3}\right)$; HRMS (ESI): $m / z \mathrm{C}_{35} \mathrm{H}_{33} \mathrm{~N}_{2} \mathrm{O}_{5}$ requires $561.2384[\mathrm{M}+\mathrm{H}]^{+}$, found $561.2402(3.23 \mathrm{ppm})$.

\section{3-[(3-Phenylisoxazol-5-yl)methyl]-3'-O-[(3-phenylisoxazol-5- yl)methyl]-5'-O-(triphenylmethyl)thymidine 13}

The crude product was purified by flash column chromatography over silica gel, eluting with dichloromethane and acetone ( 0 to $2 \%$ ), to give the title compound as an off-white solid (87\%).

$R_{\mathrm{f}} 0.70$ (DCM, Acetone, $\left.98: 2\right) ;{ }^{1} \mathrm{H}$ NMR: $\delta 7.81-7.73(5 \mathrm{H}, \mathrm{m}$, ArH), 7.62 (1H, s, H6), 7.49-7.20 (20H, m, ArH), $6.57(1 \mathrm{H}, \mathrm{s}$, $\mathrm{CH}=\mathrm{CO}$ iso $), 6.51(1 \mathrm{H}, \mathrm{s}, \mathrm{CH}=\mathrm{CO}$ iso $), 6.44-6.37\left(1 \mathrm{H}, \mathrm{m}, \mathrm{H}^{\prime}\right)$, $5.31\left(2 \mathrm{H}, \mathrm{m}, \mathrm{NCH}_{2}\right), 4.65\left(1 \mathrm{H}, \mathrm{d},{ }^{2} J 13.6, \mathrm{OCH}_{2}\right), 4.58\left(1 \mathrm{H}, \mathrm{d},{ }^{2} J\right.$ 13.6, $\left.\mathrm{OCH}_{2}\right), 4.40-4.33\left(1 \mathrm{H}, \mathrm{m}, \mathrm{H} 3^{\prime}\right), 4.21-4.15\left(1 \mathrm{H}, \mathrm{m}, \mathrm{H} 4^{\prime}\right), 3.51$ $\left(1 \mathrm{H}, \mathrm{dd},{ }^{2} J 10.9,{ }^{3} J 3.3, \mathrm{H} 5^{\prime}\right), 3.35\left(1 \mathrm{H}, \mathrm{dd},{ }^{2} J 10.7,{ }^{3} J 2.7, \mathrm{H} 5^{\prime}\right)$, 2.61-2.52 (1H, m, H2'), 2.32-2.20 (1H, m, H2'), $1.54\left(3 \mathrm{H}, \mathrm{s}, \mathrm{CH}_{3}\right)$; ${ }^{13} \mathrm{C}$ NMR: $\delta 169.0(\mathrm{C}=\mathrm{N}$ iso $), 167.7(\mathrm{C}=\mathrm{N}$ iso $), 162.7\left(\mathrm{C}_{2}\right), 162.5$ $(2 \times \mathrm{CO}=\mathrm{CH}$ iso $), 150.5\left(\mathrm{C}_{4}\right), 143.2(\mathrm{ArC}), 134.0\left(\mathrm{C}_{6}\right),[130.2$, 129.3, 129.0, 128.8, 128.7, 128.6, 128.1, 127.5, 126.9] (ArC), 110.6 $\left(\mathrm{C}_{5}\right), 101.3(2 \times \mathrm{CH}=\mathrm{CO}$ iso $), 87.6\left(\mathrm{CPh}_{3}\right), 85.6\left(\mathrm{C}_{1^{\prime}}\right), 83.9\left(\mathrm{C}_{4^{\prime}}\right)$, $80.0\left(\mathrm{C}_{3^{\prime}}\right), 63.7\left(\mathrm{C}_{5^{\prime}}\right), 62.3\left(\mathrm{OCH}_{2}\right), 38.0\left(\mathrm{C}_{2^{\prime}}\right), 36.2\left(\mathrm{NCH}_{2}\right), 12.6$ $\left(\mathrm{CH}_{3}\right)$; HRMS (ESI): $m / z \quad \mathrm{C}_{49} \mathrm{H}_{42} \mathrm{~N}_{4} \mathrm{O}_{7}$ requires $798.3048\left[\mathrm{M}^{*}\right]^{+}$, found $798.3105(7.16 \mathrm{ppm})$.

\section{3-[3-[(Naphthyl)isoxazol-5-yl]methyl]-3'-O-[3-[(naphthyl)isoxazol- 5-yl]methyl]-5'-O-(triphenylmethyl)thymidine 14}

The crude product was purified by flash column chromatography over silica gel, eluting with dichloromethane and acetone ( 0 to $2 \%)$, to give the title compound as an off-white solid $(86 \%)$.

$R_{\mathrm{f}} 0.58$ (DCM, Acetone, $\left.98: 2\right) ;{ }^{1} \mathrm{H}$ NMR: $\delta 8.43-8.29(2 \mathrm{H}, \mathrm{m}$, ArH), 7.99-7.84 (4H, m, ArH), 7.70-7.17 (24H, m, H6, ArH), 6.59 $(1 \mathrm{H}, \mathrm{s}, \mathrm{CH}=\mathrm{CO}$ iso $), 6.52(1 \mathrm{H}, \mathrm{s}, \mathrm{CH}=\mathrm{CO}$ iso $), 6.49-6.42(1 \mathrm{H}$, m, $\left.\mathrm{H}^{\prime}\right), 5.38\left(2 \mathrm{H}, \mathrm{s}, \mathrm{NCH}_{2}\right), 4.72\left(1 \mathrm{H}, \mathrm{d},{ }^{2} J 13.4, \mathrm{OCH}_{2}\right), 4.64$ 
$\left(1 \mathrm{H}, \mathrm{d},{ }^{2} J\right.$ 13.4, $\left.\mathrm{OCH}_{2}\right), 4.49-4.39\left(1 \mathrm{H}, \mathrm{m}, \mathrm{H} 3{ }^{\prime}\right), 4.26-4.18(1 \mathrm{H}$, $\left.\mathrm{m}, \mathrm{H} 4^{\prime}\right), 3.54\left(1 \mathrm{H}, \mathrm{dd},{ }^{2} J 10.7,{ }^{3} J 3.0, \mathrm{H} 5^{\prime}\right), 3.38\left(1 \mathrm{H}, \mathrm{dd},{ }^{2} J 10.7\right.$, ${ }^{3} J$ 2.7, H5'), 2.67-2.56 (1H, m, H2'), 2.38-2.26 (1H, m, H2'), 1.56 (3H, s, $\left.\mathrm{CH}_{3}\right) ;{ }^{13} \mathrm{C} \mathrm{NMR}: \delta 168.3(\mathrm{C}=\mathrm{N}$ iso $), 167.1(\mathrm{C}=\mathrm{N}$ iso $), 162.7$ $\left(\mathrm{C}_{2}\right), 162.6(2 \times \mathrm{CO}=\mathrm{CH}$ iso $), 150.5\left(\mathrm{C}_{4}\right), 143.3(\mathrm{ArC}), 134.1\left(\mathrm{C}_{6}\right)$, [133.8, 131.0, 113.4, 130.2, 128.8, 128.6, 128.5, 128.4, 128.1, 127.9, $127.5,127.2,127.0,126.8,126.5,126.4,126.2,125.8,125.6,125.2$, 125.1] (ArC), 110.6 ( $\left.\mathrm{C}_{5}\right), 104.7(2 \times \mathrm{CH}=\mathrm{CO}$ iso $), 87.6\left(\mathrm{CPh}_{3}\right), 85.6$ $\left(\mathrm{C}_{1^{\prime}}\right), 83.9\left(\mathrm{C}_{4^{\prime}}\right), 80.1\left(\mathrm{C}_{3^{\prime}}\right), 63.7\left(\mathrm{C}_{5^{\prime}}\right), 62.3\left(\mathrm{OCH}_{2}\right), 38.0\left(\mathrm{C}_{2^{\prime}}\right), 36.4$ $\left(\mathrm{NCH}_{2}\right), 12.6\left(\mathrm{CH}_{3}\right)$; HRMS (ESI): $m / z \mathrm{C}_{57} \mathrm{H}_{46} \mathrm{~N}_{4} \mathrm{NaO}_{7}$ requires $921.3259[\mathrm{M}+\mathrm{Na}]^{+}$, found $921.3282(2.52 \mathrm{ppm})$.

\section{3-(2-Propynyl)-3'-O-(2-propynyl)thymidine 12}

The crude product was purified by flash column chromatography over silica gel, eluting with dichloromethane and methanol ( 0 to $5 \%$ ), to give the title compound as a off-white foam ( $94 \%)$.

$R_{\mathrm{f}} 0.39$ (DCM, MeOH, 95: 5); ${ }^{1} \mathrm{H}$ NMR: $\delta 7.43(1 \mathrm{H}, \mathrm{s}, \mathrm{H} 6)$, $6.14\left(1 \mathrm{H}, \mathrm{t},{ }^{3} J\right.$ 7.5, H1'), $4.72\left(2 \mathrm{H}, \mathrm{d},{ }^{4} J \mathrm{~J} 2.4, \mathrm{NCH}_{2}\right), 4.47-4.40$ $\left(1 \mathrm{H}, \mathrm{m}, \mathrm{H}^{\prime}\right), 4.28-4.05\left(4 \mathrm{H}, \mathrm{m}, \mathrm{H} 4, \mathrm{OCH}_{2}, \mathrm{OH}\right), 3.97(1 \mathrm{H}$, $\mathrm{dd},{ }^{2} J 12.5,{ }^{3} J$ 2.7, $\left.\mathrm{H} 5^{\prime}\right), 3.85\left(1 \mathrm{H}, \mathrm{dd},{ }^{2} J 12.5,{ }^{3} J 3.0, \mathrm{H} 5^{\prime}\right)$, $2.49\left(1 \mathrm{H}, \mathrm{t},{ }^{4} \mathrm{~J} 2.4, \mathrm{OCH}_{2} \mathrm{C} \equiv \mathrm{CH}\right), 2.47-2.36\left(2 \mathrm{H}, \mathrm{m}, \mathrm{H} 2^{\prime}\right), 2.17$ $\left(1 \mathrm{H}, \mathrm{t},{ }^{4} \mathrm{~J} 2.4, \mathrm{NCH}_{2} \mathrm{C} \equiv \mathrm{CH}\right), 1.96\left(3 \mathrm{H}, \mathrm{s}, \mathrm{CH}_{3}\right),{ }^{13} \mathrm{C} \mathrm{NMR}: \delta$ $162.6\left(\mathrm{C}_{2}\right), 150.2\left(\mathrm{C}_{4}\right), 135.5\left(\mathrm{C}_{6}\right), 110.5\left(\mathrm{C}_{5}\right), 88.0\left(\mathrm{C}_{1^{\prime}}\right), 84.9$ $\left(\mathrm{C}_{4^{\prime}}\right), 79.1\left(\mathrm{OCH}_{2} \mathrm{C} \equiv \mathrm{CH}\right), 78.4\left(\mathrm{C}_{3^{\prime}}\right), 78.0\left(\mathrm{NCH}_{2} \mathrm{C} \equiv \mathrm{CH}\right), 75.2$ $\left(\mathrm{OCH}_{2} \mathrm{C} \equiv \mathrm{CH}\right), 70.8\left(\mathrm{NCH}_{2} \mathrm{C} \equiv \mathrm{CH}\right), 62.6\left(\mathrm{C}_{5^{\prime}}\right), 56.9\left(\mathrm{OCH}_{2}\right), 38.0$ $\left(\mathrm{C}_{2^{\prime}}\right), 30.5\left(\mathrm{NCH}_{2}\right), 13.2\left(\mathrm{CH}_{3}\right)$; HRMS (ESI): $m / z \mathrm{C}_{16} \mathrm{H}_{18} \mathrm{KN}_{2} \mathrm{O}_{5}$ requires $357.0847[\mathrm{M}+\mathrm{K}]^{+}$, found $357.0853(1.46 \mathrm{ppm})$.

\section{3-[(3-Phenylisoxazol-5-yl)methyl]-3'-O-[(3-phenylisoxazol-5- yl)methyl|thymidine 15}

The crude product was purified by flash column chromatography over silica gel, eluting with dichloromethane and methanol $(98: 2)$, to give the title compound as an off-white solid (80\%).

$R_{\mathrm{f}} 0.35$ (DCM, MeOH, $\left.98: 2\right) ;{ }^{1} \mathrm{H}$ NMR: $\delta$ 7.78-7.64 $(4 \mathrm{H}, \mathrm{m}$, $\mathrm{ArH}), 7.44-7.28(7 \mathrm{H}, \mathrm{m}, \mathrm{H} 6, \mathrm{ArH}), 6.52(1 \mathrm{H}, \mathrm{s}, \mathrm{CH}=\mathrm{CO}$ iso $)$, $6.49(1 \mathrm{H}, \mathrm{s}, \mathrm{CH}=\mathrm{CO}$ iso $), 6.11\left(1 \mathrm{H}, \mathrm{t},{ }^{3} J 7.7, \mathrm{H} 1^{\prime}\right), 5.22(2 \mathrm{H}, \mathrm{s}$, $\left.\mathrm{NCH}_{2}\right), 4.63\left(1 \mathrm{H}, \mathrm{d},{ }^{2} J 13.4, \mathrm{OCH}_{2}\right), 4.58\left(1 \mathrm{H}, \mathrm{d},{ }^{2} J 13.4, \mathrm{OCH}_{2}\right)$, 4.36-4.28 (1H, m, H3'), 4.12-4.06 (1H, m, H4'), $3.88(1 \mathrm{H}$, dd, ${ }^{2} J 11.6,{ }^{3} J$ 2.4, H5'), 3.74 (1H, br d, $\left.{ }^{2} J 11.5, \mathrm{H} 5^{\prime}\right), 2.52-2.25$ $\left(3 \mathrm{H}, \mathrm{m}, \mathrm{H} 2^{\prime}, \mathrm{OH}\right), 1.88\left(3 \mathrm{H}, \mathrm{s}, \mathrm{CH}_{3}\right) ;{ }^{13} \mathrm{C} \mathrm{NMR}: \delta 168.0(\mathrm{C}=\mathrm{N}$ iso $), 166.6(\mathrm{C}=\mathrm{N}$ iso $), 161.6\left(\mathrm{C}_{2}\right), 161.5(2 \times \mathrm{CO}=\mathrm{CH}$ iso $), 149.5$ $\left(\mathrm{C}_{4}\right), 134.3\left(\mathrm{C}_{6}\right),[129.2,129.0,128.1,128.0,127.9,127.8,127.7$, 127.6, 127.3, 127.2, 126.5, 126.1, 125.8] (ArC), $109.4\left(\mathrm{C}_{5}\right), 100.6$ $(\mathrm{CH}=\mathrm{CO}$ iso $), 100.4(\mathrm{CH}=\mathrm{CO}$ iso $), 86.8\left(\mathrm{C}_{1^{\prime}}\right), 84.0\left(\mathrm{C}_{4^{\prime}}\right), 78.7$ $\left(\mathrm{C}_{3^{\prime}}\right), 61.7\left(\mathrm{C}_{5^{\prime}}\right), 61.4\left(\mathrm{OCH}_{2}\right), 36.2\left(\mathrm{C}_{2^{\prime}}\right), 35.2\left(\mathrm{NCH}_{2}\right), 12.2\left(\mathrm{CH}_{3}\right)$; HRMS (ESI): $m / z \mathrm{C}_{30} \mathrm{H}_{29} \mathrm{~N}_{4} \mathrm{O}_{7}$ requires $557.2031[\mathrm{M}+\mathrm{H}]^{+}$, found 557.2051 (3.71 ppm).

\section{3-[3-[(Naphthyl)isoxazol-5-yl]methyl]-3'-O-[3-[(naphthyl)isoxazol- 5-yl]methyl]thymidine 16}

The crude product was purified by flash column chromatography over silica gel, eluting with dichloromethane and acetone $(9: 1)$, to give the title compound as an off-white solid (78\%).

$R_{\mathrm{f}} 0.44$ (DCM, MeOH, $\left.95: 5\right) ;{ }^{1} \mathrm{H}$ NMR: $\delta 8.40-8.31(2 \mathrm{H}, \mathrm{m}$, ArH), 7.99-7.83 (4H, m, ArH), 7.73-7.44 (9H, m, H6, ArH), 6.60 $(1 \mathrm{H}, \mathrm{s}, \mathrm{CH}=\mathrm{CO}$ iso $), 6.58(1 \mathrm{H}, \mathrm{s}, \mathrm{CH}=\mathrm{CO}$ iso $), 6.26-6.19(1 \mathrm{H}$, m, H1'), $5.37\left(2 \mathrm{H}, \mathrm{s}, \mathrm{NCH}_{2}\right), 4.76\left(1 \mathrm{H}, \mathrm{d},{ }^{2} J 13.6, \mathrm{OCH}_{2}\right), 4.70$ $\left(1 \mathrm{H}, \mathrm{d},{ }^{2} J\right.$ 13.6, $\left.\mathrm{OCH}_{2}\right), 4.48-4.39\left(1 \mathrm{H}, \mathrm{m}, \mathrm{H} 3^{\prime}\right), 4.22-4.14(1 \mathrm{H}$ $\left.\mathrm{m}, \mathrm{H} 4^{\prime}\right), 3.79-3.40\left(2 \mathrm{H}, \mathrm{m}, \mathrm{H} 5^{\prime}\right), 2.68(1 \mathrm{H}, \mathrm{s}, \mathrm{OH}), 2.54-2.33(2 \mathrm{H}$, m, H2') $1.95\left(3 \mathrm{H}, \mathrm{s}, \mathrm{CH}_{3}\right) ;{ }^{13} \mathrm{C}$ NMR: $\delta 168.3(\mathrm{C}=\mathrm{N}$ iso $), 167.0$ $(\mathrm{C}=\mathrm{N}$ iso $), 162.7\left(\mathrm{C}_{2}\right), 150.6\left(\mathrm{C}_{4}\right), 135.4(2 \times \mathrm{CO}=\mathrm{CH}$ iso $), 133.8$ $\left(\mathrm{C}_{6}\right),[131.0,130.4,130.2,128.6,128.4,127.8,127.2,127.0,126.7$, 126.4, 126.2, 125.7, 125.5, 125.2, 125.1] (ArC), $110.4\left(\mathrm{C}_{5}\right), 104.8$ $(\mathrm{CH}=\mathrm{CO}$ iso $), 104.7(\mathrm{CH}=\mathrm{CO}$ iso $), 87.8\left(\mathrm{C}_{1^{\prime}}\right), 85.0\left(\mathrm{C}_{4^{\prime}}\right), 79.9$ $\left(\mathrm{C}_{3^{\prime}}\right), 67.9\left(\mathrm{C}_{5^{\prime}}\right), 62.5\left(\mathrm{OCH}_{2}\right), 37.3\left(\mathrm{C}_{2^{\prime}}\right), 36.3\left(\mathrm{NCH}_{2}\right), 13.2\left(\mathrm{CH}_{3}\right)$; HRMS (ESI): $m / z \mathrm{C}_{38} \mathrm{H}_{33} \mathrm{~N}_{4} \mathrm{O}_{7}$ requires $658.2375[\mathrm{M}+\mathrm{H}]^{+}$, found 658.2388 (1.84 ppm).

\section{3'-Thymidylic acid, 2-cyanoethyl 4-(2-propynyl-1-yloxy)butyl ester 20}

To a round bottom flask containing a solution of alkynyl alcohol $17^{19}(72 \mathrm{mg}, 0.562 \mathrm{mmol})$ in dry acetonitrile $(10 \mathrm{~mL})$ was added, under argon, BMT (BMT = 5-benzylmercaptotetrazole, $215 \mathrm{mg}$, $1.119 \mathrm{mmol})$ followed by dT-CE phosphoramidite $18(500 \mathrm{mg}$, $0.671 \mathrm{mmol}$ ). The mixture was stirred for $0.5 \mathrm{~h}$ at RT after which analysis by TLC (hexane-EtOAc, $1: 1$ ) indicated complete reaction. The mixture was cooled to $0{ }^{\circ} \mathrm{C}$ in order to add drop by drop the solution of iodine $\left(0.1 \mathrm{M}\right.$ in $\mathrm{THF} /$ pyridine $/ \mathrm{H}_{2} \mathrm{O}$ $78: 20: 2 / \mathrm{mL})$. The resulting mixture was stirred for $0.5 \mathrm{~h}$ at $0{ }^{\circ} \mathrm{C}$, and after addition of ethyl acetate $(20 \mathrm{~mL})$, washed with a saturated solution of sodium thiosulfate. The organic layer was dried over anhydrous sodium sulfate and the solvent was removed under reduced pressure. The crude product $(551 \mathrm{mg})$ was dissolved in a solution of DCA $3.5 \%$ in DCM $(10 \mathrm{~mL})$, and was stirred for $0.5 \mathrm{~h}$ at RT. The reaction mixture was washed with a saturated solution of sodium hydrogen carbonate until the aqueous layer became neutral. The organic layer was dried over anhydrous sodium sulfate and the solvent was removed under reduced pressure. The crude product was purified by flash column chromatography over silica gel using dichloromethane and methanol ( 0 to $10 \%)$ as eluant to give the title compound as a yellow oil $(53 \%)$.

$R_{\mathrm{f}} 0.50$ (DCM, MeOH, $\left.95: 5\right) ;{ }^{1} \mathrm{H}$ NMR: $\delta 9.71(1 \mathrm{H}$, br s, NH), $7.55(1 \mathrm{H}, \mathrm{s}, \mathrm{H} 6), 6.24\left(1 \mathrm{H}, \mathrm{t},{ }^{3} J\right.$ 7.2, H1'), $5.17\left(1 \mathrm{H}, \mathrm{br} \mathrm{s}, \mathrm{H}^{\prime}\right), 4.35$ $4.10\left(7 \mathrm{H}, \mathrm{m}, \mathrm{H}^{\prime}, \mathrm{OCH}_{2}\right.$ iso, $\left.2 \times \mathrm{POCH}_{2}\right), 3.88\left(1 \mathrm{H}\right.$, br s, $\left.\mathrm{H} 5^{\prime}\right)$, $3.76\left(1 \mathrm{H}\right.$, br s, OH), $3.56\left(2 \mathrm{H}, \mathrm{t},{ }^{3} \mathrm{~J} 5.6, \mathrm{OC}_{2} \mathrm{CH}_{2}\right), 2.82\left(2 \mathrm{H}, \mathrm{t},{ }^{3} \mathrm{~J}\right.$ 5.6, $\left.\mathrm{CH}_{2} \mathrm{CN}\right), 2.60-2.35\left(3 \mathrm{H}, \mathrm{m}, \mathrm{H} 2^{\prime}, \mathrm{C} \equiv \mathrm{CH}\right), 1.90\left(3 \mathrm{H}, \mathrm{s}, \mathrm{CH}_{3}\right)$, 1.86-1.63 (4H, m, $\left.\mathrm{OCH}_{2} \mathrm{C}_{2} \mathrm{C}_{\underline{H}} \mathrm{CH}_{2} \mathrm{O}\right) \cdot{ }^{13} \mathrm{C} \mathrm{NMR}: \delta 164.1\left(\mathrm{C}_{2}\right)$, $150.6\left(\mathrm{C}_{4}\right), 136.5\left(\mathrm{C}_{6}\right), 116.7(\mathrm{C} \equiv \mathrm{N}), 111.3\left(\mathrm{C}_{5}\right), 85.8\left(\mathrm{C}_{1^{\prime}}\right), 85.7$ $\left(\mathrm{C}_{4^{\prime}}\right), 79.8(\mathrm{C} \equiv \mathrm{CH}), 78.4\left(\mathrm{C}_{3^{\prime}}\right), 74.5(\mathrm{C} \equiv \mathrm{CH}), 69.2\left(\mathrm{OCH}_{2} \mathrm{CH}_{2}\right)$, $68.7\left(\mathrm{POCH}_{2}\right), 62.3\left(\mathrm{POCH}_{2}\right), 61.8\left(\mathrm{C}_{5^{\prime}}\right), 58.1\left(\mathrm{OCH}_{2}\right.$ iso $), 38.5$ $\left(\mathrm{C}_{2^{\prime}}\right), 27.2\left(\mathrm{OCH}_{2} \mathrm{CH}_{2} \mathrm{CH}_{2} \mathrm{CH}_{2} \mathrm{O}\right), 25.4\left(\mathrm{OCH}_{2} \mathrm{CH}_{2} \mathrm{CH}_{2} \mathrm{CH}_{2} \mathrm{O}\right)$, $19.7\left(\mathrm{CH}_{2} \mathrm{C} \equiv \mathrm{N}\right), 12.5\left(\mathrm{CH}_{3}\right) ;{ }^{31} \mathrm{P}$ NMR: $\delta-2.6$; HRMS (ESI): $m / z \mathrm{C}_{20} \mathrm{H}_{28} \mathrm{KN}_{3} \mathrm{O}_{9} \mathrm{P}$ requires $524.1195[\mathrm{M}+\mathrm{K}]^{+}$, found 524.1196 (0.27 ppm).

\section{3'-Thymidylic acid, 2-cyanoethyl 4-[I(3-phenylisoxazole-5- yl)methyl]oxy]butyl ester 21}

The crude product was purified by flash column chromatography over silica gel, eluting with dichloromethane and methanol (0 to $5 \%$ ), to give the title compound as a yellow oil $(77 \%)$.

$R_{\mathrm{f}} 0.54$ (DCM, MeOH, $\left.95: 5\right) ;{ }^{1} \mathrm{H}$ NMR: $\delta 9.45(1 \mathrm{H}$, br s, NH), 7.82-7.75 (2H, m, ArH), 7.51 (1H, s, H6), 7.48-7.41 (3H, m, ArH), $6.58(1 \mathrm{H}, \mathrm{s}, \mathrm{CH}=\mathrm{CO}$ iso $), 6.22\left(1 \mathrm{H}, \mathrm{t},{ }^{3} J\right.$ 7.5, $\left.\mathrm{H}^{\prime}\right), 5.19-5.12(1 \mathrm{H}$, 
$\left.\mathrm{m}, \mathrm{H} 3^{\prime}\right), 4.63\left(2 \mathrm{H}, \mathrm{m}, \mathrm{OCH}_{2}\right.$ iso $), 4.33-4.11\left(5 \mathrm{H}, \mathrm{m}, \mathrm{H} 4^{\prime}, 2 \times\right.$ $\left.\mathrm{POCH}_{2}\right), 3.87\left(2 \mathrm{H}\right.$, br s, H5'), $3.61\left(2 \mathrm{H}, \mathrm{t},{ }^{3} \mathrm{~J} 6.0, \mathrm{OC}_{\underline{2}} \mathrm{CH}_{2}\right), 2.78$ $\left(2 \mathrm{H}, \mathrm{t},{ }^{3} \mathrm{~J} 6.0, \mathrm{CH}_{2} \mathrm{CN}\right), 2.59-2.40\left(2 \mathrm{H}, \mathrm{m}, \mathrm{H} 2^{\prime}\right), 2.17$ (1H, br s, $\mathrm{OH}), 1.92-1.69\left(7 \mathrm{H}, \mathrm{m}, \mathrm{CH}_{3}, \mathrm{OCH}_{2} \mathrm{CH}_{2} \mathrm{CH}_{2} \mathrm{CH}_{2} \mathrm{O}\right){ }^{13} \mathrm{C} \mathrm{NMR}: \delta$ $169.8(\mathrm{C}=\mathrm{N}$ iso $), 163.9\left(\mathrm{C}_{2}\right), 162.5(\mathrm{CO}=\mathrm{CH}), 150.5\left(\mathrm{C}_{4}\right), 136.5$ $\left(\mathrm{C}_{6}\right), 130.2(\mathrm{ArCH}), 129.0(\mathrm{ArCH}), 128.8(\mathrm{ArC}), 126.8(\mathrm{ArCH})$, $116.6(\mathrm{C} \equiv \mathrm{N}), 111.3\left(\mathrm{C}_{5}\right), 101.2(\mathrm{CH}=\mathrm{CO}$ iso $), 86.0\left(\mathrm{C}_{1^{\prime}}\right), 85.6$ $\left(\mathrm{C}_{4^{\prime}}\right), 78.4\left(\mathrm{C}_{3^{\prime}}\right), 70.5\left(\mathrm{OCH}_{2} \mathrm{CH}_{2}\right), 68.7\left(\mathrm{POCH}_{2}\right), 63.7\left(\mathrm{OCH}_{2}\right.$ iso $)$, $62.2\left(\mathrm{POCH}_{2}\right), 62.0\left(\mathrm{C}_{5^{\prime}}\right), 38.4\left(\mathrm{C}_{2^{\prime}}\right), 27.2\left(\mathrm{OCH}_{2} \mathrm{CH}_{2} \mathrm{CH}_{2} \mathrm{CH}_{2} \mathrm{O}\right)$, $25.6\left(\mathrm{OCH}_{2} \mathrm{CH}_{2} \mathrm{CH}_{2} \mathrm{CH}_{2} \mathrm{O}\right), 19.8\left(\mathrm{CH}_{2} \mathrm{C} \equiv \mathrm{N}\right), 12.5\left(\mathrm{CH}_{3}\right) ;{ }^{31} \mathrm{P}$ NMR: $\delta-2.5$; HRMS (ESI): $m / z \mathrm{C}_{27} \mathrm{H}_{35} \mathrm{~N}_{4} \mathrm{O}_{10} \mathrm{P}$ requires 303.1040 $[\mathrm{M}+2 \mathrm{H}]^{2+}$, found $303.1041(0.46 \mathrm{ppm})$.

\section{3'-Thymidylic acid, 2-cyanoethyl 4-[|[3-[(1-naphthyl)isoxazole-5- yl]methyl]oxy]butyl ester 22}

The crude product was purified by flash column chromatography over silica gel, eluting with dichloromethane and methanol (0 to $5 \%$, to give the title compound as a yellow oil $(72 \%)$.

$R_{\mathrm{f}} 0.57$ (DCM, MeOH, 95:5); ${ }^{1} \mathrm{H}$ NMR: $\delta 8.91(1 \mathrm{H}$, br s, NH), 8.38-8.31 (1H, m, ArH), 7.99-7.88 (2H, m, ArH), 7.73-7.67 (1H, m, ArH), 7.59-7.50 (3H, m, ArH), 7.43 (1H, s, H6), 6.59 $(1 \mathrm{H}, \mathrm{s}, \mathrm{CH}=\mathrm{CO}$ iso $), 6.19\left(1 \mathrm{H}, \mathrm{t},{ }^{3} J\right.$ 7.2, $\left.\mathrm{H} 1^{\prime}\right), 5.19-5.11(1 \mathrm{H}$, $\left.\mathrm{m}, \mathrm{H}^{\prime}\right), 4.71\left(2 \mathrm{H}, \mathrm{m}, \mathrm{OCH}_{2}\right.$ iso), 4.34-4.13 (5H, m, H4', $2 \times$ $\left.\mathrm{POCH}_{2}\right), 3.88\left(2 \mathrm{H}\right.$, br s, H5'), $3.67\left(2 \mathrm{H}, \mathrm{t},{ }^{3} \mathrm{~J} 6.0, \mathrm{OC}_{2} \mathrm{CH}_{2}\right)$, $3.18\left(1 \mathrm{H}\right.$, br s, OH), $2.77\left(2 \mathrm{H}, \mathrm{t},{ }^{3} \mathrm{~J} 6.0, \mathrm{CH}_{2} \mathrm{CN}\right), 2.58-2.40$ (2H, m, H2'), 1.95-1.70 (7H, m, $\left.\mathrm{CH}_{3}, \mathrm{OCH}_{2} \mathrm{CH}_{2} \mathrm{C}_{\underline{H}} \mathrm{CH}_{2} \mathrm{O}\right) ;{ }^{13} \mathrm{C}$ NMR: $\delta 169.1(\mathrm{C}=\mathrm{N}$ iso $), 163.6\left(\mathrm{C}_{2}\right), 162.6(C \mathrm{O}=\mathrm{CH}), 150.4$ $\left(\mathrm{C}_{4}\right), 136.5\left(\mathrm{C}_{6}\right),[130.9,130.4,128.6,127.8,127.1,126.6,126.4$, 125.5, 125.2] (ArC), $116.6(\mathrm{C} \equiv \mathrm{N}), 111.3\left(\mathrm{C}_{5}\right), 104.5(\mathrm{CH}=\mathrm{CO}$ iso), $86.2\left(\mathrm{C}_{1^{\prime}}\right), 85.6\left(\mathrm{C}_{4^{\prime}}\right), 78.3\left(\mathrm{C}_{3^{\prime}}\right), 70.6\left(\mathrm{OCH}_{2} \mathrm{CH}_{2}\right), 68.6$ $\left(\mathrm{POCH}_{2}\right), 63.7\left(\mathrm{OCH}_{2}\right.$ iso $), 62.2\left(\mathrm{POCH}_{2}\right), 62.0\left(\mathrm{C}_{5^{\prime}}\right), 38.4$ $\left(\mathrm{C}_{2^{\prime}}\right), 27.2\left(\mathrm{OCH}_{2} \mathrm{CH}_{2} \mathrm{CH}_{2} \mathrm{CH}_{2} \mathrm{O}\right), 25.6\left(\mathrm{OCH}_{2} \mathrm{CH}_{2} \mathrm{CH}_{2} \mathrm{CH}_{2} \mathrm{O}\right)$, $19.8\left(\mathrm{CH}_{2} \mathrm{C} \equiv \mathrm{N}\right), 12.5\left(\mathrm{CH}_{3}\right) ;{ }^{31} \mathrm{P}$ NMR: $\delta-2.4$; HRMS (ESI): $m / z \mathrm{C}_{31} \mathrm{H}_{37} \mathrm{~N}_{4} \mathrm{O}_{10} \mathrm{P}$ requires $328.1118[\mathrm{M}+2 \mathrm{H}]^{2+}$, found 328.1119 (0.40 ppm).

\section{Acknowledgements}

Financial support from the Science Foundation of Ireland (Programme code 05/PICA/B838) is gratefully acknowledged.

\section{References}

1 B.-H. Kim and Y.-S. Lee, PCT Int. Pat., WO 20030185772003.

2 L. S. Jeong and J. A. Lee, Antiviral Chem. Chemother., 2004, 15, 235.

3 N. A. Brown, Expert Opin. Invest. Drugs, 2009, 18, 709.

4 M. F. A. Adamo and R. Pergoli, Curr. Org. Chem., 2008, 12, 1544.

5 B. L. Deng, T. L. Hartman, R. W. Buckheit, C. Pannecouque, E. De Clercq and M. Cushman, J. Med. Chem., 2006, 49, 5316.

6 K. Itoh, H. Sakamaki, N. Nakazato, A. Horiuchi, E. Horn and C. A. Horiuchi, Synthesis, 2005, 3541.

7 Y.-S. Lee and B.-H. Kim, Bioorg. Med. Chem. Lett., 2002, 12, 1395.

8 J. Cao and X. Huang, J. Comb. Chem., 2008, 10, 526.

9 H.-J. Gi, Y. Xiang, R. F. Schinazi and K. Zhao, J. Org. Chem., 1997, 62, 88 .

10 G. Giglio, A. Napoli, A. Leggio, A. Liguori, A. Procopio, C. Siciliano and G. Sindona, Synth. Commun., 1996, 26, 4211.

11 Y. Xiang, H.-J. Gi, D. Niu, R. F. Schinazi and K. Zhao, J. Org. Chem., 1997, 62, 7430.

12 Y.-S. Lee, S. M. Park and B. H. Kim, Bioorg. Med. Chem. Lett., 2009, 19, 1126.

13 E. Coutouli-Argyropoulou, P. Lianis, M. Mitakou, A. Giannoulis and J. Nowak, Tetrahedron, 2006, 62, 1494.

14 D. R. Adams, C. Perez, M. Maillard, J.-C. Florent, M. Evers, Y. Henin, S. Litvak, L. Litvak, C. Monneret and D. S. Grierson, J. Med. Chem., 1997, 40, 1550.

15 J. P. Mogensen, S. M. Roberts, A. N. Bowler, C. Thomsen and L. J. S. Knutsen, Bioorg. Med. Chem. Lett., 1998, 8, 1767.

16 A. Plant, P. Thompson and D. M. Williams, J. Org. Chem., 2009, 74, 4870.

17 S. J. Kim, J. Y. Lee and B.-H. Kim, Bioorg. Med. Chem. Lett., 1998, 8, 1313.

18 J. R. Kong, S. K. Kim, B. J. Moon, S. J. Kim and B. H. Kim, Nucleosides, Nucleotides Nucleic Acids, 2001, 20, 1751.

19 I. Singh, J. S. Vyle and F. Heaney, Chem. Commun., 2009, 3276.

20 K. Gutsmiedl, C. T. Wirges, V. Ehmke and T. Carell, Org. Lett., 2009, 11, 2405.

21 F. Amblard, J. H. Cho and R. F. Schinazi, Chem. Rev., 2009, 109, 4207.

22 C. Chan, R. P. C. Cousins and B. Cox, PCT Int. Pat., WO 9938877 1999.

23 I. Singh and F. Heaney, Org. Biomol. Chem., 2010, DOI: 10.1039/ b918463e.

24 A. M. Michelson and A. R. Todd, J. Chem. Soc., 1953, 951.

25 S. Liekens, A. Bronckaers, A.-I. Hernandez, E.-M. Priego, E. Casanova, M.-J. Camarasa, M.-J. Perez-Perez and J. Balzarini, Mol. Pharmacol., 2006, 70, 501

26 A. Rosowsky, R. M. Ruprecht and V. C. Solan, Nucleosides, Nucleotides Nucleic Acids, 1989, 8, 491.

27 A. Hassner and K. M. L. Rai, Synthesis, 1989, 57.

28 S. Grecian and V. V. Fokin, Angew. Chem., Int. Ed., 2008, 47, 8285

29 R. Lucas, R. Zerrouki, R. Granet, P. Krausz and Y. Champavier, Tetrahedron, 2008, 64, 5467.

30 R. Lucas, V. Neto, A. HadJ Bouazza, R. Zerrouki, R. Granet, P. Krausz and Y. Champavier, Tetrahedron Lett., 2008, 49, 1004. 